\title{
Transcranial brain stimulation (TMS and tDCS) for post-stroke aphasia rehabilitation
}

\author{
Controversies
}

\author{
Lucia Iracema Zanotto de Mendonça ${ }^{1}$
}

\begin{abstract}
Transcranial brain stimulation (TS) techniques have been investigated for use in the rehabilitation of post-stroke aphasia. According to previous reports, functional recovery by the left hemisphere improves recovery from aphasia, when compared with right hemisphere participation. TS has been applied to stimulate the activity of the left hemisphere or to inhibit homotopic areas in the right hemisphere. Various factors can interfere with the brain's response to TS, including the size and location of the lesion, the time elapsed since the causal event, and individual differences in the hemispheric language dominance pattern. The following questions are discussed in the present article: [a] Is inhibition of the right hemisphere truly beneficial?; [b] Is the transference of the language network to the left hemisphere truly desirable in all patients?; [c] Is the use of TS during the poststroke subacute phase truly appropriate? Different patterns of neuroplasticity must occur in post-stroke aphasia. Key words: aphasia, transcranial magnetic stimulation, rehabilitation.
\end{abstract}

\section{ESTIMULAÇÃO CEREBRAL TRANSCRANIANA (EMT E ETCC) NA REABILITAÇÃO DE AFASIA: CONTROVÉRSIAS}

RESUMO. As técnicas de estimulação cerebral transcraniana (ET) têm sido estudadas como recurso na reabilitação da afasia resultante de acidente vascular cerebral. Tem sido apontado que melhor recuperação da afasia ocorre quando o hemisfério esquerdo reassume a função da linguagem, quando comparado à participação do hemisfério direito. A ET pode estimular a atividade do hemisfério esquerdo ou inibir a atividade de áreas homotópicas do hemisfério direito. Vários fatores podem interferir na resposta à ET, como o tamanho e local da lesão, o tempo decorrido do evento causal e diferenças individuais no padrão de dominância hemisférica para a linguagem. Este artigo discute as seguintes questões: [a] Realmente é benéfico inibir o hemisfério direito? [b] Realmente é desejável a transferência para a esquerda da função da linguagem em todos os casos? [c] Realmente é adequada a aplicação da TS na fase subaguda pós AVC? Diferentes padrões de reorganização cerebral devem ocorrer frente à presença de afasia decorrente de AVC.

Palavras-chave: afasia, estimulação magnética transcraniana, reabilitação.

\section{INTRODUCTION}

Alogical diseases, especially stroke, and re-
covery differs between patients.
In healthy individuals, language is a com-
plex function that includes the participation
of multiple brain areas from both hemi-
spheres. For this reason, language is particu-
larly vulnerable to brain injury. Language ex-
hibits lateralization, and the left hemisphere shows dominance in linguistic skills for $96 \%$ of healthy, right-handed individuals. ${ }^{1}$ Nevertheless, human communication is based on a set of phonological, semantic, discursive and pragmatic features, which depend on the integration between left and right hemispheres and interaction with other cognitive functions. These data suggest that the right hemisphere plays a specific role in language. The pattern of hemispheric language dominance

${ }^{1} \mathrm{MD}$, PhD. Mestre e Doutora em Neurologia - Faculdade de Medicina da Universidade de São Paulo, SP, Brazil.

Lucia I. Z. de Mendonça. Rua André Dreifus, 264 - 01252-010 São Paulo SP - Brazil. E-mail: lucia.iracema@terra.com.br

Disclosure: The authors report no conflicts of interest.

Received February 02, 2014. Accepted in final form April 03, 2014 
is related to laterality ${ }^{1}$ and literacy. ${ }^{2}$ Functional neuroimaging has detected changes in the pattern of brain activation in bi/multilingualism ${ }^{3,4}$ and as a function of age. ${ }^{5,6}$ Thus, the brain circuits associated with language vary based on the life experiences of an individual, and this individualization may influence the reorganization of the neural network that occurs after brain injury.

Two TS methods have been used for the rehabilitation of patients with aphasia, including transcranial directcurrent stimulation (tDCS) and transcranial magnetic stimulation (TMS). Both methods are considered safe. ${ }^{7,8}$

Left and right hemispheres can participate in aphasia recovery. Better recovery has been associated with the restoration of function by the left hemisfhere. ${ }^{9}$

Models of interhemispheric competition have been described for motor and sensory systems. By extending this concept to the language domain, the intact right hemisphere may exert inhibitory influences on the lesioned left hemisphere and interfere with the reacquisition of efficient language processing through lefthemisphere cortical networks(Figure 1A). There is some support for the hypothesis of reciprocal transcallosal inhibition in language networks. ${ }^{10}$

TS may exert excitatory or inhibitory effects on the underlying brain tissue. Therefore, TS has been used to favor recruitment of left-hemispheric language networks, increasing the activity of the left hemisphere (ipsilateral to the lesion) or disrupting interhemispheric inhibition by downregulating the activity of the right hemisphere (contralateral to the lesion) (Figure $1 \mathrm{~B}$ and $1 \mathrm{C}$ ).

However, some authors have argued that the right hemisphere is important for recuperation at least in some patients ${ }^{9}$ and that homotopic areas are not necessarily homologous areas. ${ }^{11}$ Their data suggest that the strategy of promoting functional recovery by the left hemisphere and of inhibiting the right hemisphere may not be effective for all patients.
The purpose of this article is to discuss TS use in the context of the possible mechanisms of network language reorganization. The controversial topics are the inhibition of homotopic areas in the right hemisphere, the use of TS in the subacute phase post-stroke, and methodological aspects of the studies. This article also highlights the need to respect individual differences in the language network prior to the lesion.

\section{POST-APHASIA FUNCTIONAL REORGANIZATION}

After damage to the left hemispheric language network, the functional recovery of aphasia can occur by activation of the perilesional area in the left hemisphere, by recruitment of residual left-hemispheric structures that may have been previously involved in language function, or by activation of the right hemisphere. ${ }^{12}$

The contribution of each brain hemisphere to recovery from aphasia is controversial. The role of the right hemisphere in language recovery and its interaction with damaged left-hemispheric structures has not been elucidated.

\section{CONTRIBUTION OF THE LEFT HEMISPHERE}

Satisfactory recovery from aphasia has been consistently associated with the restoration of left-hemispheric functions. Neurofunctional studies have correlated left lateralization and activation with improved language ability, ${ }^{13,14}$ suggesting that the preservation or restoration of the left hemispheric language network is important for recovery from aphasia.

However, the activation of right-side homotopic areas was also found in individuals who exhibited satisfactory recovery. ${ }^{11}$

\section{CONTRIBUTION OF THE RIGHT HEMISPHERE}

Evidence indicates that the right hemisphere contributes to recovery from aphasia. Children who suffered
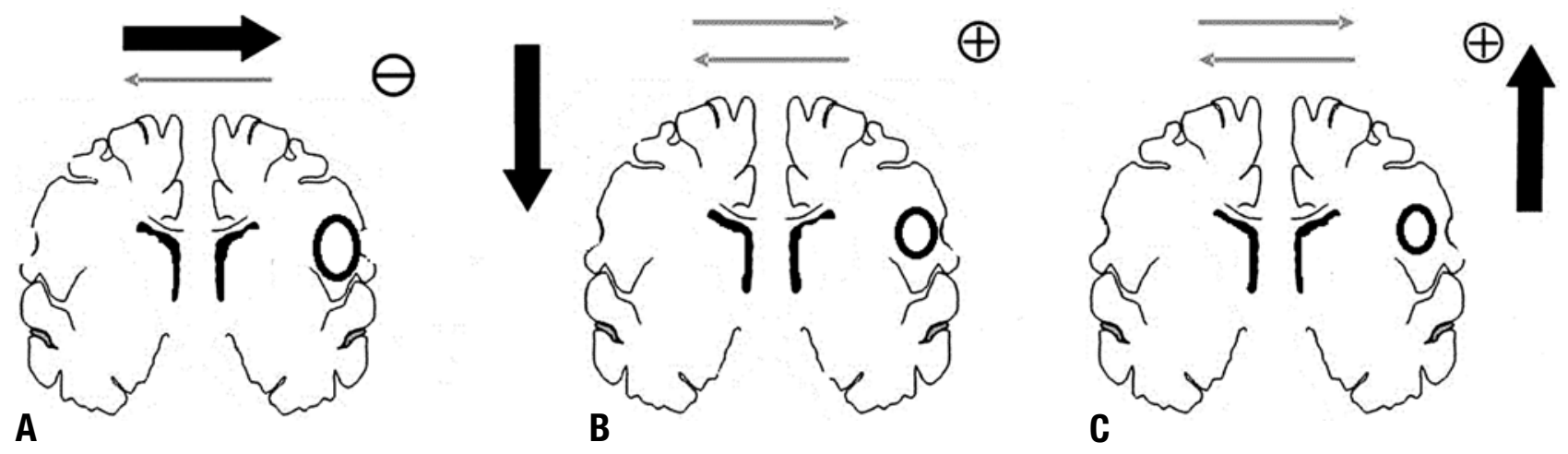

Figure 1. $[A]$ Intact hemisphere may exert high inhibitory influences on the lesioned hemisphere. $[B]$ Inhibitory TS contralateral to the lesion rebalances the interhemispheric interaction. [C] Excitatory TS ipsilateral to the lesion rebalances the interhemispheric interaction. 
extensive damage to the left hemisphere or who were subjected to hemispherectomy exhibited significant language recovery. ${ }^{15}$ In adults who displayed satisfactory recovery from aphasia following brain damage to the left hemisphere, subsequent injury of the right hemisphere was able to cause new functional deterioration. ${ }^{16}$ Functional neuroimaging and TMS have shown the transfer of language functions to the right hemisphere in patients with slowly progressing left hemisphere tumors. ${ }^{17}$

The right hemisphere participates in the recovery from aphasia through the activation of homotopic areas, which are analogous in location to the language areas of the left hemisphere. ${ }^{11}$ These areas constitute a useful compensatory network for speech disorders, even if they are computationally less efficient.

However, the activation of these brain areas can be dysfunctional. For instance, right-side homotopic areas may be related to other features of communication and thus result in maladaptive recovery. The contribution of the right hemisphere to recovery from aphasia might be due to its participation in executive function, attention and memory, rather than through direct language restoration. ${ }^{18}$

Some studies have found a relationship between activation of the right hemisphere and aphasia improvement. ${ }^{9}$ Other studies suggest that the right-hemispheric shift as a mechanism of post-stroke recovery in adults is an ineffective way for language function recovery. ${ }^{19}$

Therefore, the functional relevance of the activation of homotopic right-hemispheric language areas remains ambiguous.

\section{INFLUENCE OF LESION SIZE AND RECOVERY TIMES}

Post-injury brain activation patterns depend on the size and extent of the lesion. The recruitment of left perilesional areas with variable involvement of righthemispheric structures occurs in small lesions in the left hemisphere. ${ }^{20}$ In addition, the participation of the right hemisphere is often significant in large lesions. ${ }^{21} \mathrm{Howev}-$ er, a significant correlation between lesion volume in the dominant hemisphere and activation of the non-dominant hemisphere has not been determined. ${ }^{14}$ In addition to the activation of left-hemispheric language regions, a robust activation in homotopic right-hemispheric regions regardless of lesion size has been observed. ${ }^{22}$

These data must be assessed as a function of recovery period. Initially, after a stroke, there may be a reallocation of language function to the right hemisphere, particularly in patients with extensive left-hemispheric injury. Over time, this recruitment diminishes and is followed by a redistribution of language processing back to the left hemisphere; however, this process is more likely to occur in patients with relatively small lesions. ${ }^{9,20}$

Changes in the activation pattern of the brain hemispheres over time suggest that the initial temporary increase in right-hemispheric activation does not necessarily reflect a functionally relevant reorganization process. Alternatively, the increased activation may be due to changes in transcallosal inhibition.

Specific participation of the right hemisphere in recovery from aphasia is possible. Activation of the left hemisphere is not reestablished in all cases, especially in individuals with large left-side lesions. Increased activation of the right inferior frontal gyrus (IFG) from the acute to the subacute phase, is associated with improved language performance. ${ }^{9}$ Recruitment of the right hemisphere during recovery from aphasia can be effective if it occurs during a critical time window poststroke and depends on the lesion's location, extent and permanence. ${ }^{23}$

In summary, the brain mechanisms involved with reorganization during recovery from aphasia are variable and depend on the size and extent of the lesion and on recovery time.

\section{TS IN APHASIA REHABILITATION}

The first studies that used TS in patients with aphasia involved cases of nonfluent aphasia due to stroke. TS techniques have also been used in cases of progressive aphasia. ${ }^{24}$ The following discussion mainly focuses on aphasia secondary to vascular brain injury because degenerative conditions progress slowly and likely result in a different brain reorganization pattern.

TS has been used alone or concomitantly with speech and language therapy; however, both favorable ${ }^{25}$ and ineffective ${ }^{26}$ results have been reported with TS alone. Thus, whether TS should be used alone or combined with additional behavioral treatment strategies remains unclear. TS may further improve aphasia symptoms by potentiating the neural signals elicited by other therapies.

\section{STIMULATION OF THE LEFT HEMISPHERE}

One aim of TS is activation of the left hemisphere.

TS has been applied to Broca's ${ }^{27-29}$ and Wernicke's ${ }^{29,30}$ areas.

TS applied in Broca's area significantly improved naming accuracy, ${ }^{27}$ semantic fluency ${ }^{28}$ and spontaneous speech, as evidenced by the ability to use connective words to establish cohesion among adjacent utterances. ${ }^{29}$

The fMRI maps obtained after TS showed increased activation of the left fronto-temporo-parietal language 
networks with a significant left-hemispheric shift compared with images taken prior to treatment. ${ }^{28}$

Differences in functional improvement have been found based on the site of TS. A significantly greater improvement in noun naming was found after stimulation of the temporal region, while verb naming significantly improved after stimulation of the frontal region. ${ }^{30}$

\section{INHIBITION OF THE RIGHT HEMISPHERE}

A large number of studies have focused on inhibition of the right hemisphere.

Researchers have particularly focused on the triangular portion of the right inferior frontal gyrus (IFG).

Several aphasia scales show improvements in naming, repetition, reaction time and oral expression and comprehension following the application of right-side inhibitory TS. ${ }^{25,31,32}$ The improvement in naming is particularly significant for action naming. ${ }^{33}$ An improvement in picture descriptions with respect to the number of narrative words and nouns, sentence length, and the use of closed class words has also been described. ${ }^{33}$

However, not all studies have reported favorable results following inhibition of the right hemisphere. Poor outcomes in naming, semantics, fluency and reaction time have also been described, ${ }^{33-36}$ suggesting that the right IFG may play an essential role in the residual language function of some patients.

Naeser et al. ${ }^{37}$ emphasized that the application of inhibitory stimulation to the right pars opercularis of the IFG (POp) impaired performance, while the same stimulation of the right pars triangularis ( $\mathrm{PTr}$ ) improved performance. ${ }^{25,37}$

Therefore, when TS is applied to the IFG, local anatomical-functional features should be taken into consideration.

Broca's area is located in the posterior IFG (pIFG) and encompasses Brodmann's areas 44 (approximately corresponding to the opercular portion of the $\mathrm{PIFG}$ ) and 45 (approximately corresponding to the triangular portion of the pIFG). The two regions of right Broca's homolog (the PTr and POp) may be functionally different and might play different roles in aphasia recovery.

Evidence suggests that the right POp plays a causal role in phonologic processing in normal subjects. ${ }^{38}$ Functional imaging studies have shown that the right and left pIFG are activated when healthy right-handed individuals make phonological word decisions ${ }^{39,40}$.

Results from fMRI demonstrate that there is a reliable increase in activation for semantic relative to phonological decisions in the anterior region of IFG (PTr), while the opposite comparison (phonological vs. se- mantic decisions) shows an area of enhanced activation within the posterior region of IFG (POp).$^{40}$

TMS can be used to temporarily interfere with neural processing in the IFG. TMS applied over the anterior IFG (PTr) significantly slowed subjects' reactions for the semantic tasks, ${ }^{40}$ while TMS of posterior IFG (POp) impaired reaction times and accuracy of phonological decision tasks. ${ }^{38}$ TMS over left, right or bilateral pIFG disrupted phonological processing to a similar degree. ${ }^{38}$

In summary, bilateral POp is related with phonologic processing and left PTr is related with semantic processing.

Wernicke's and Broca's areas are linked by the arcuate fasciculus, which is integrated into the superior longitudinal fasciculus. This dorsal stream is related with phonologic processes. ${ }^{41}$ The uncinate fasciculus connects the anterior and middle temporal lobe and the ventrolateral prefrontal cortex. This ventral pathway is related with the semantic process. ${ }^{41}$

The diffusion tensor imaging (DTI)-based tractography method allows visualization of white matter pathways in vivo. Using DTI images, Kaplan et al. ${ }^{42}$ studied the pathways related to subregions of Broca's area - PTr and POp. Almost no fiber tracts were visible between PTr and the dorsal pathway in the left and right hemispheres. In contrast to PTr, 8/8 subjects showed robust fiber tracts between POp and the arcuate fasciculus/ superior longitudinal fasciculus in the left hemisphere, and $5 / 8$ participants in the right hemisphere.

Therefore, there are functional differences between the subregions of the IFG. Dorsal stream phonologic processes, in which the POp is involved, may be less lateralized, compared with word-level semantic processes associated with the ventral stream and the PTr. Many, but not all, of these areas are homologous in function. ${ }^{43}$

Turkeltaub $^{43}$ reviewed the literature for fMRI or PET studies employing language tasks in patients with chronic aphasia after stroke and healthy controls, using a validated, quantitative neuroimaging meta-analysis method in order to assess mechanisms of adaptation in aphasia. In aphasic subjects, a bilateral distribution of activation included spared areas of the normal left-hemispheric language network, left-hemispheric areas outside the normal network, and right-hemispheric areas that mirrored the left-hemispheric network in controls.

The greatest likelihood for activation was in the IFG, although bilaterally. In general, the right IFG was more reliably recruited when the left inferior frontal cortex was lesioned, but this effect differed between subregions of the IFG.

The right POp was homotopic and functionally ho- 
mologous to the control subjects' left POp. The right PTr was homotopic to a left-hemispheric control site but was not functionally homologous. Two different areas of the right PTr were recruited, depending on the lesion location, and the function in both areas was unlike that of the normal left PTr.

Thus, the application of inhibitory stimulation to the right $\mathrm{POp}$ impairs performance because it interferes with right POp normal function. Although the studies show that stimulation of the right PTr improves performance, ${ }^{25,37}$ Turkeltaub's results suggest a pattern of adaptation after lesioning in the left-hemispheric language networks that involves variation in the mechanisms of right hemisphere recruitment which depends on lesion location. ${ }^{36,43}$

Therefore, the reorganization of the language network following brain injury varies. Different compensatory mechanisms are allocated depending on which part of the network is disrupted. The activation of the right hemisphere is not necessarily maladaptive. Language recovery after stroke may integrate left- and righthemispheric brain regions to different degrees during the recovery process. Inhibition of the right hemisphere might hamper language recovery.

Some authors have sought the best point for application of inhibitory TS to the right hemisphere. The stimulation site can be determined by using fMRI during a language task; TS is applied to an area homologous to the site with the greatest activation from the fMRI results $^{27}$. Based on the fMRI results, inhibitory TMS could also be applied to the left hemisphere ${ }^{44}$. An exploratory phase delivered to different sites in the right frontal lobe, preceded and followed by a language task, can select the optimal area for stimulation. ${ }^{45}$

Individualized TS is also controversial. The interference of TS on the possible utilization of a functional architecture by the right hemisphere after left-hemispheric injury is unclear. This fact may be evidenced by Turkeltaub's et al. case. ${ }^{46}$ A woman with chronic nonfluent aphasia showed improved naming after inhibitory TMS of the right hemisphere. fMRI confirmed a local reduction in activity at the TMS target without the expected increase in activity in the corresponding left-hemispheric area. Three months after TMS, the patient suffered a right-hemispheric ischemic stroke that resulted in a worsening of aphasia.

\section{PATIENT-SPECIFIC DIFFERENCES IN THE LANGUAGE NETWORK}

The particular features of the cerebral language network should be taken into consideration when TS techniques are used. For instance, the participation of the right and left hemispheres varies as a function of laterality.

Heiss et al. ${ }^{47}$ used inhibitory TMS in the contralesional IFG together with speech and language therapy in subacute post-stroke aphasia patients. A greater level of recovery in language function on global aphasia test scores was found in TMS-treated right-handed patients compared with sham-treated right-handed patients. Language activation patterns assessed with PET showed a shift of activation to the ipsilesional hemisphere in TMS-treated patients, while sham-treated patients consolidated network activity in the contralesional hemisphere. However, the therapeutic efficiency was doubtful in two cases of left-handed aphasics, although no deterioration of language performance was observed. Both patients exhibited a very small interhemispheric shift.

The individual brain organization language patterns in older adults and illiterate or multilingual individuals have not been taken into consideration in previous TS studies.

\section{USE OF TS IN SUBACUTE POST-STROKE APHASIA}

Most studies have used TS in individuals with chronic aphasia. The findings of these investigations cannot be extrapolated to acute or subacute aphasia because the neural adaptation in aphasia changes over time.

The results from the small number of studies on subacute post-stroke aphasia are contradictory. TS was coupled with speech and language therapy in all of these studies.

Stimulation of the affected left hemisphere during the early post-stroke rehabilitation period did not produce statistically significant differences between patients who received $t D C S$ or sham $t D C S$ with regards to naming accuracy and naming time. ${ }^{48}$

Small group differences in the degree of recovery were found between patients receiving TMS that inhibited the right-hemispheric homolog of Broca's area and control participants. ${ }^{49}$ However, follow-up revealed that severely aphasic rTMS patients demonstrated significantly greater improvement in repetition, compared with patients receiving sham stimulation. This result suggests that inhibitory TS applied to the right frontal language homolog is not effective for all post-stroke aphasia patients, although it may benefit a select group of patients. ${ }^{49}$

Other studies revealed significant clinical improvements in naming, comprehension, token tests and writing using inhibitory TS of the right-hemispheric Broca homolog. ${ }^{50-52}$ Results of positron emission tomography (PET) in these analyses showed increased activation 
in the left hemisphere after treatment, compared with sham-treated patients, and a shift toward the right hemisphere in the control group. This change in laterality indices may ${ }^{52}$ or may not ${ }^{50}$ be related to clinical improvement. Recovery from aphasia due to the restoration of left-hemispheric functioning as shown by a shift in the activation pattern toward the left on PET would be a promising sign. However, this type of transference is not necessarily correlated with clinical improvement and therefore the true significance of this process during the subacute stage of brain injury is not clear.

Winhuisen et al. ${ }^{53,54}$ studied the extent to which the activation of the right IFG is essential for language performance in subacute poststroke aphasia, at different times. They used TMS stimulation to interfere with the function of right and left IFG on a semantic task. At 2 weeks after left hemispheric stroke, ${ }^{53} \mathrm{PET}$ activations of the IFG were observed to the left ( 3 patients) and bilaterally (8 patients). Right IFG stimulation increased reaction time latency or error rate on the semantic task in 5 patients, indicating that in some poststroke aphasics, right IFG activation is essential for residual language function. To test whether the right IFG remained essential for language performance, they reexamined 9 patients, 8 weeks after stroke. ${ }^{54}$ Language function had improved in all patients. At this timepoint, PET activations of the IFG were observed to the left (2 patients) and bilaterally (7 patients). TMS over the left IFG interfered with the language performance in all patients, indicating that the left IFG remained essential. Stimulation over the right IFG interfered with the language performance in 2 patients. Two patients with positive TMS effects over the right side in the initial study did not show these effects at follow-up. The authors suggest that restoration of the left hemisphere network seems to be more effective for aphasia recuperation.

Research on the neuroplasticity following brain injury in animals has shown that commencing training soon after injury ${ }^{55-58}$ and a high degree of stimulation ${ }^{56,59,60}$ hindered recovery by exaggerating excitotoxicity in the vulnerable perilesional tissue. These data suggest the presence of time- and intensity-dependent brain vulnerability.

Caution is required when extrapolating experimental animal data to clinical conditions. The majority of current neuroplasticity knowledge concerns the primary motor, sensory, auditory and visual cortices, which are functionally quite different from language. The ideal time to start a therapeutic intervention in rats was 14 days post-lesion..$^{59}$ Rats typically have a lifespan of 2-3 years; thus, 14 days post-lesion may be equivalent to a longer therapeutic window in humans. In humans, re- organization of the brain circuits and clinical recovery occur spontaneously in 2-3 months. ${ }^{61}$

Evidence from basic science contradicts the general and accepted clinical evidence. The aphasia treatment literature shows that therapy should be started as soon as possible $e^{62}$ and that intensive treatment over short periods of time is better than less-intensive regimens over a longer period of time. ${ }^{63}$

The early onset of rehabilitation therapy might prevent disuse and dysfunctional plasticity.

Basic science has shown that neural circuits that are not used for some time become inactive and suffer degradation. ${ }^{64}$ Thus, if disuse hinders recovery, then therapy might preserve the cortical function representation.

The interaction between the brain's adaptation to damage (spontaneous recovery) and therapy over time is another factor that should be taken into consideration. A brain that one may attempt to reorganize with rehabilitative training is one that is being, and likely already has been, driven to reorganize by compensatory behavioral changes. ${ }^{65}$ In this regard, early commencement of rehabilitation therapy should improve recovery.

In an attempt to combine clinical and experimental data, previous work began rehabilitation therapy at a lower intensity which was then increased gradually over time. ${ }^{58}$

The aforementioned treatment considerations account for the introduction of speech and language therapy. The following issues should be considered when using TS for acute or subacute aphasia:

[A] The method may produce overstimulation at a critical stage of brain injury evolution. The neural signal sent by speech and language therapy is likely less intense than the signal produced by TS, although may help to maintain the cortical function representation.

[B] Otherwise, TS may force the left hemisphere to reassume the language function, which appears to produce better results. Enhanced activation of the right hemisphere can be observed within 2 weeks after stroke and may return to control levels after 1 year, whereas lefthemispheric activity increases gradually over months to years. ${ }^{9}$ These data suggest that the early application of TS is beneficial. However, the functional meaning of the changes in activation between brain hemispheres remains unknown. Previous studies ${ }^{9,21,43,53}$ suggest that right-hemispheric regions may beneficially contribute to the recovery of a subset of patients; thus, it is important to identify which patients should receive early TS.

Further investigation of TS as a treatment during the acute or subacute phases of post-stroke aphasia is needed. 


\section{METHODOLOGICAL ASPECTS}

Methodological questions should be mentioned.

Control groups are lacking in some studies. . $5,28,29,33,44^{2}$ The participant's performance is analyzed before and after TS application series. The placebo effect could explain the improvement observed.

Language performance is not assessed using a formal test battery in some studies. Naming, ${ }^{25,32,48,66}$ word repetition, ${ }^{67}$ semantic decision, ${ }^{19}$ spontaneous elicited speech $^{29}$ are considered outcome measures after TS.

The authors do not report change of test material, e.g. for picture naming, before and after TS. Consequently, a learning effect could be possible.

Some studies highlight the need for more thorough research.

Elsner et al. ${ }^{8}$ assessed the effects of tDCS with respect to improving aphasia in patients after stroke. These authors included only randomized controlled trials and randomized controlled cross-over trials. No studies used a formal outcome measure for measuring functional communication, and correct picture naming was used as a surrogate for aphasia. There was no evidence that tDCS enhanced speech and language therapy outcomes. The authors concluded that there is no evidence for the effectiveness of tDCS (anodal tDCS, cathodal tDCS) versus control (sham tDCS) in post-stroke aphasia. However, it appears that cathodal tDCS of the non-lesioned hemisphere may be the most promising approach.

Wong and Tsang ${ }^{7}$ performed an evidence-based review of the literature as to the effectiveness of TMS on post-stroke aphasia. The controlled trials showed a positive effect of TMS, with or without conventional rehabilitation, on post-stroke aphasia when compared with sham or conventional rehabilitation alone. However, the authors emphasized that concerns over the methodology of the selected studies warrant a larger-scale, multicenter, well-designed randomized controlled trial involving different phases and types of aphasia before recommending rTMS as a complementary treatment for post-stroke aphasia.

\section{CONCLUSION}

The clinical use of TS methods in post-stroke aphasia rehabilitation remains controversial.

Before the clinical use of TS can be recommended, the following pertinent questions must be answered:

a) Is inhibition of the right hemisphere truly beneficial? Behavioral evidence confirms that compensatory reorganization occurs within the right hemisphere after the original stroke.
Homotopic brain areas are not necessarily homologous in their function. The involvement of certain righthemispheric areas may support recovery in a subset of patients, ${ }^{46}$ especially in cases of large left-side lesions; however, there may not be a correlation between lesion volume in the dominant hemisphere and activation in the non-dominant hemispheric counterparts. ${ }^{14,22}$

b) Is the transference of the language network to the left side truly desirable in all cases? The brain adaptation that occurs in post-stroke aphasia constitutes a dynamic and progressive process. Increased activation of the left hemisphere after treatment, as quantified by PET, may not correlate with clinical improvement. ${ }^{31}$ The functional meaning of the changes in activation between the brain hemispheres at different stages of clinical evolution in patients is unknown. The meaning of righthemispheric activation in patients with small lesions that retain some original level of activation is not clear.

c) Is the application of TS during the post-stroke subacute phase truly appropriate? Some studies on neuroplasticity point to the presence of time- and intensity-dependent brain vulnerability. Applying TS too soon after the occurrence of brain injury and with a high degree of stimulation have been shown to hinder recovery.

The application of TS in the post-stroke subacute phase can produce overstimulation and excitotoxicity, both of which are detrimental for recovery. There are no reports in the literature that compare the long-term progression of individuals subjected to TS in the subacute and chronic phases.

d) Can TS protocols not consider the variables age, gender, laterality, literacy and bi/multilinguism? These variables are linked to particular language network and possibly different mechanisms of reorganization after lesion. $\mathrm{Pa}-$ tient-specific factors result in differential recruitment from individual to individual. ${ }^{43}$

Aphasia recovery is associated with a complex pattern of brain reorganization, ${ }^{68}$ involving both ipsilateral and contralateral brain regions, modulated by lesion size and site, time post-onset, training type, and language task. ${ }^{69}$ The differences in recovery mechanisms may be dependent on which part of the network is disrupted..$^{43}$ Factors such as language aspect affected, the degree of language lateralization, age, gender and literacy should be included in TS protocols. The use of TS for recovery from post-stroke aphasia is highly promising; however, future studies with larger patient groups are needed before recommending this method for clinical use. 


\section{REFERENCES}

1. Rasmussen T, Milner B. The role of early left-brain injury in determining lateralization of cerebral speech functions. Ann N Y Acad Sci 1977;299: 355-369

2. Lecours AR, Mehler J, Parente MA, et al. Illiteracy and brain damage. 3: A contribution to the study of speech and language disorders in illiterates with unilateral brain damage (initial testing). Neuropsychologia 1988;26:575-589.

3. Zou L, Abutalebi J, Zinszer B, et al. Second language experience modulates functional brain network for the native language production in bimodal bilinguals. Neuroimage 2012;62:1367-1375.

4. Bloch $\mathrm{C}$, Kaiser A, Kuenzli E, et al. The age of second language acquisition determines the variability in activation elicited by narration in three languages in Broca's and Wernicke's area. Neuropsychologia 2009;47:625-633

5. Destrieux C, Hommet C, Domengie F, et al. Influence of age on the dynamics of $\mathrm{fMRl}$ activations during a semantic fluency task. J Neuroradiol 2012;39:158-166.

6. Scherer LC, Fonseca RP, Giroux F, et al. Neurofunctional (re)organization underlying narrative discourse processing in aging: evidence from fNIRS. Brain Lang 2012;121:174-184.

7. Wong IS, Tsang HW. A review on the effectiveness of repetitive transcranial magnetic stimulation (rTMS) on post-stroke aphasia. Rev Neurosci 2013;24:105-114

8. Elsner B, Kugler J, Pohl M, Mehrholz J. Transcranial direct current stimulation (tDCS) for improving aphasia in patients after stroke. Cochrane Database Syst Rev 2013; 6:CD009760.

9. Saur D, Lange R, Baumgaertner A, Schraknepper V, Willmes K, Rijntjes $\mathrm{M}$ et al. Dynamics of language reorganization after stroke. Brain 2006;129:1371-1384.

10. Thiel A, Schumacher B, Wienhard K, et al. Direct demonstration of transcallosal disinhibition in language networks. J Cereb Blood Flow Metab 2006;26:1122-1127.

11. Calvert GA, Brammer MJ, Morris RG, Williams SC, King N, Matthews $\mathrm{PM}$. Using $\mathrm{fMRI}$ to study recovery from acquired dysphasia. Brain Lang 2000;71:391-399.

12. Heiss WD, Thiel A. A proposed regional hierarchy in recovery of poststroke aphasia. Brain Lang 2006; 98:118-123.

13. Karbe H, Thiel A, Weber-Luxenburger G, Herholz K, Kessler J, Heiss WD. Brain plasticity in poststroke aphasia: what is the contribution of the right hemisphere? Brain Lang 1998;64:215-230.

14. van Oers CA, Vink M, van Zandvoort MJ, et al. Contribution of the left and right inferior frontal gyrus in recovery from aphasia. A functional MR study in stroke patients with preserved hemodynamic responsiveness. Neuroimage 2010;49:885-893.

15. Vargha-Khadem F, Carr LJ, Isaacs E, Brett E, Adams C, Mishkin M. Onset of speech after left hemispherectomy in a nine-year-old boy. Brain 1997;120:159-182.

16. Basso A, Gardelli M, Grassi MP, Mariotti M. The role of the right hemisphere in recovery from aphasia. Two case studies. Cortex 1989; 25: 555-566.

17. Thiel A, Habedank B, Herholz K, et al. From the left to the right: How the brain compensates progressive loss of language function. Brain Lang 2006;98:57-65.

18. Crosson B, Moore AB, Gopinath K, et al. Role of the right and left hemispheres in recovery of function during treatment of intention in aphasia. J Cogn Neurosci 2005;17:392-406.

19. Szaflarski JP, Allendorfer JB, Banks C, Vannest J, Holland SK. Recovered vs. not- recovered from post-stroke aphasia: the contributions from the dominant and non-dominant hemispheres. Restor Neurol Neurosci 2013;31:347-360.

20. Hillis AE. Aphasia: progress in the last quarter of a century. Neurology 2007;69:200-213.

21. Heiss WD, Thiel A. A proposed regional hierarchy in recovery of poststroke aphasia. Brain Lang 2006;98:118-123.

22. Rosen HJ, Petersen SE, Linenweber MR, et al. Neural correlates of recovery from aphasia after damage to left inferior frontal cortex. Neurology 2000;55:1883-1894.

23. Anglade $\mathrm{C}$, Thiel $\mathrm{A}$, Ansaldo Al. The complementary role of the cerebra hemispheres in recovery from aphasia after stroke: a critical review of literature. Brain Inj 2014;28:138-145.
24. Wang J, Wu D, Chen $Y$, Yuan Y, Zhang M. Effects of transcranial direct current stimulation on language improvement and cortical activation in nonfluent variant primary progressive aphasia. Neurosci Lett 2013;549:29-33.

25. Naeser MA, Martin PI, Nicholas M, et al. Improved picture naming in chronic aphasia after TMS to part of right Broca's area: an open-protocol study. Brain Lang 2005;93:95-105.

26. Volpato C, Cavinato M, Piccione F, Garzon M, Meneghello F, Birbaumer N. Transcranial direct current stimulation (tDCS) of Broca's area in chronic aphasia: a controlled outcome study. Behav Brain Res 2013; 247:211-216.

27. Baker JM, Rorden C, Fridriksson J. Using transcranial direct-current stimulation to treat stroke patients with aphasia. Stroke 2010;41:12291236.

28. Szaflarski JP, Vannest J, Wu SW, DiFrancesco MW, Banks C, Gilbert DL. Excitatory repetitive transcranial magnetic stimulation induces improvements in chronic post-stroke aphasia. Med Sci Monit 2011;17:CR132-9.

29. Marangolo P, Fiori V, Campana S, et al. Something to talk about: enhancement of linguistic cohesion through tdCS in chronic non fluent aphasia. Neuropsychologia 2014;53:246-256.

30. Fiori V, Cipollari S, Di Paola M, Razzano C, Caltagirone C, Marangolo P. tDCS stimulation segregates words in the brain: evidence from aphasia. Front Hum Neurosci 2013;7:269.

31. Weiduschat N, Thiel A, Rubi-Fessen I, et al. Effects of repetitive transcranial magnetic stimulation in aphasic stroke: a randomized controlled pilot study. Stroke 2011;42:409-415.

32. Barwood $\mathrm{CH}$, Murdoch BE, Whelan BM, et al. The effects of low frequency Repetitive Transcranial Magnetic Stimulation (rTMS) and sham condition rTMS on behavioural language in chronic non-fluent aphasia: Short term outcomes. NeuroRehabilitation 2011;28:113-128.

33. Hamilton RH, Sanders L, Benson J, et al. Stimulating conversation: enhancement of elicited propositional speech in a patient with chronic non-fluent aphasia following transcranial magnetic stimulation. Brain Lang 2010;113:45-50.

34. Winhuisen L, Thiel A, Schumacher B, et al. Role of the contralateral inferior frontal gyrus in recovery of language function in poststroke aphasia: a combined repetitive transcranial magnetic stimulation and positron emission tomography study. Stroke 2005;36:1759-1763.

35. Martin PI, Naeser MA, Ho M, et al. Research with transcranial magnetic stimulation in the treatment of aphasia. Curr Neurol Neurosci Rep 2009;9:451-458.

36. Martin PI, Naeser MA, Ho M, et al. Overt naming fMRI pre- and postTMS: Two nonfluent aphasia patients, with and without improved naming post-TMS. Brain Lang 2009;111:20-35

37. Naeser MA, Martin PI, Treglia E, et al. Research with rTMS in the treatment of aphasia. Restor Neurol Neurosci 2010;28:511-529.

38. Hartwigsen G, Price CJ, Baumgaertner A, et al.The right posterior inferior frontal gyrus contributes to phonological word decisions in the healthy brain: evidence from dual-site TMS. Neuropsychologia 2010;48:3155-3163.

39. Poldrack RA, Wagner AD, Prull MW, Desmond J E, Glover GH, Gabrieli JD. Functional specialization for semantic and phonological processing in the left inferior prefrontal cortex. Neuroimage 1999;10:15-35.

40. Devlin JT, Matthews PM, Rushworth MF. Semantic processing in the left inferior prefrontal cortex: A combined functional magnetic resonance imaging and transcranial magnetic stimulation study. J Cogn Neurosci 2003;15:71-84.

41. Saur D, Kreher BW, Schnell S, et al. Ventral and dorsal pathways for language. Proc Natl Acad Sci 2008;105:18035-18040.

42. Kaplan E, Naeser MA, Martin PI, et al.Horizontal portion of arcuate fasciculus fibers track to pars opercularis, not pars triangularis, in right and left hemispheres: a DTI study. Neuroimage 2010;52:436-444.

43. Turkeltaub PE, Messing, S, Norise C, Hamilton RH. Are networks for residual language function and recovery consistent across aphasic patients? Neurology 2011;76:1726-1734

44. Kakuda W, Abo M, Kaito N, Watanabe M, Senoo A. Functional MRIbased therapeutic rTMS strategy for aphasic stroke patients: a case series pilot study. Int J Neurosci 2010;120:60-66.

45. Garcia G, Norise C, Faseyitan O, Naeser MA, Hamilton RH. Utilizing repetitive transcranial magnetic stimulation to improve language func- 
tion in stroke patients with chronic non-fluent aphasia. J Vis Exp 2013; 77:e50228.

46. Turkeltaub PE, Coslett HB, Thomas AL, et al. The right hemisphere is not unitary in its role in aphasia recovery. Cortex 2012;48:1179-1186.

47. Heiss WD, Hartmann A, Rubi-Fessen I, et al. Noninvasive brain stimulation for treatment of right- and left-handed poststroke aphasics. Cerebrovasc Dis 2013;36:363-372.

48. Polanowska KE, Lesniak MM, Seniów JB, Czepiel W, Czlonkowska A. Anodal transcranial direct current stimulation in early rehabilitation of patients with post-stroke non-fluent aphasia: a randomized, double-blind, sham-controlled pilot study. Restor Neurol Neurosci 2013;31:761-771.

49. Seniów J, Waldowski K, Lesniak M, Iwanski S, Czepiel W, Czlonkowska A. Transcranial magnetic stimulation combined with speech and language training in early aphasia rehabilitation: a randomized double-blind controlled pilot study. Top Stroke Rehabil 2013;20:250-261.

50. Weiduschat N, Thiel A, Rubi-Fessen I, et al. Effects of repetitive transcranial magnetic stimulation in aphasic stroke: a randomized controlled pilot study. Stroke 2011;42:409-415.

51. Heiss WD, Hartmann A, Rubi-Fessen I, et al. Noninvasive brain stimulation for treatment of right- and left-handed poststroke aphasics. Cerebrovasc Dis 2013;36:363-372.

52. Thiel A, Hartmann A, Rubi-Fessen I, et al. Effects of noninvasive brain stimulation on language networks and recovery in early poststroke aphasia. Stroke 2013;44:2240-2246.

53. Winhuisen L, Thiel A, Schumacher B, et al. Role of the contralateral inferior frontal gyrus in recovery of language function in poststroke aphasia: a combined repetitive transcranial magnetic stimulation and positron emission tomography study. Stroke 2005;36:1759-1763.

54. Winhuisen L, Thiel A, Schumacher B, et al. The right inferior frontal gyrus and poststroke aphasia: a follow-up investigation. Stroke 2007:38:1286-1292.

55. Humm JL, Kozlowski DA, James DC, Gotts JE, Schallert T. Use-dependent exacerbation of brain damage occurs during an early post-lesion vulnerable period. Brain Res 1998;783:286-292.

56. Farrell R, Evans S, Corbett D. Environmental enrichment enhances re- covery of function but exacerbates ischemic cell death. Neuroscience 2001; 107:585-592

57. Kleim JA, Jones TA, Schallert T. Motor enrichment and the induction of plasticity before or after brain injury. Neurochem Res 2003;28:17571769.

58. Woodlee MT, Schallert T. The interplay between behavior and neurodegeneration in rat models of Parkinson's disease and stroke. Restor Neurol Neurosci 2004;22:153-161.

59. Schallert T, Kozlowski DA, Humm JL, Cocke RR. Use-dependent structural events in recovery of function. Adv Neurol 1997;73:229-238.

60. DeBow SB, McKenna JE, Kolb B, Colbourne F. Immediate constraintinduced movement therapy causes local hyperthermia that exacerbates cerebral cortical injury in rats. Can J Physiol Pharmacol 2004;82:231-237.

61. Lendrem W, Lincoln NB. Spontaneous recovery of language in patients with aphasia between 4 and 34 weeks after stroke. J Neurol Neurosurg Psychiatry 1985;48:743-748.

62. Robey RR. A meta-analysis of clinical outcomes in the treatment of aphasia. J Speech Lang Hear Res 1998;41:172-187.

63. Bhogal SK, Teasell RW, Foley NC, Speechley MR. Community reintegration after stroke. Top Stroke Rehabil 2003;10:107-129.

64. Hubel DH, Wiesel TN. Binocular teraction in strate cortex of kittens reared with artificial squint. J Neurophysiol 1965;28:1041-1059.

65. Kleim JA, Jones TA. Principles of experience-dependent neural plasticity: implications for rehabilitation after brain damage. J Speech Lang Hear Res 2008;51:S225-239.

66. Baker JM, Rorden C, Fridriksson J. Using transcranial direct-current stimulation to treat stroke patients with aphasia. Stroke 2010;41:1229-1236.

67. Abo M, Kakuda W. Neuroimaging and neurorehabilitation for aphasia. Brain Nerve 2010;62:141-149.

68. Hamilton RH, Chrysikou EG, Coslett B. Mechanisms of aphasia recovery after stroke and the role of noninvasive brain stimulation. Brain Lang 2011;118:40-50

69. Cappa SF. The neural basis of aphasia rehabilitation: evidence from neuroimaging and neurostimulation. Neuropsychol Rehabil 2011;21:742754. 